\title{
Factores psicológicos asociados a la formación y al rendimiento en judokas de elite
}

Psychological factors associated with training and performance in elite judokas

Andrés Robles-Rodríguez 1

Centro Universitario San Isidoro, adscrito a la Universidad Pablo de Olavide, Sevilla, España

Manuel Tomás Abad-Robles [i]

Universidad de Huelva

José Robles-Rodríguez [i]

Universidad de Huelva

Francisco Javier Giménez ${ }^{\circ}$

Universidad de Huelva

jfuentes@uhu.es

\begin{abstract}
Resumen
El proceso de formación de los deportistas desde que se inician hasta que llegan a la elite va a depender de aspectos de tipo biológico, psicológico, perceptivo-cognitivo y social. El objetivo principal de este estudio ha sido analizar la importancia que los factores psicológicos tienen en el proceso formativo recorrido por los judokas de elite españoles que han representado a España en los Juegos Olímpicos celebrados en el siglo XXI (Sidney 2000, Atenas 2004, Pekín 2008, Londres 2012, y Rio 2016). Este periodo abarca cinco Juegos Olímpicos y 25 judokas ( 15 mujeres y 10 hombres). La muestra final a los que se pudo acceder fue de 20 deportistas ( $80 \%$ de la población total) distribuidos de la siguiente forma: mujeres ( $n=12,60$ $\%$ ) y hombres $(n=8,40 \%)$. El instrumento de investigación fue una entrevista semiestructurada. Las conclusiones de este trabajo destacan, en primer lugar, la gran importancia de los aspectos psicológicos en el proceso formativo según la opinión de los propios deportistas. En este sentido, los contenidos psicológicos más importantes para llegar a la elite, según los entrevistados, son la perseverancia, la constancia, la capacidad de sacrifico y la autoconfianza. También la motivación es valorada de forma muy necesaria, sobre todo en los comienzos para conseguir hábitos de práctica y evitar abandonos prematuros.
\end{abstract}

Palabras claves: formación deportiva, judo, juegos olímpicos, aspectos psicológicos.

\begin{abstract}
The process of training athletes from the moment they start until they reach the elite will depend on biological, psychological, perceptive-cognitive and social aspects. The main aim of this study has been to analyze the importance that psychological factors have in the formative process of the Spanish elite judokas that have represented Spain in the Olympic Games held in the 21 st century (Sydney 2000, Athens 2004, Beijing 2008, London 2012, and Rio 2016). This period covers five Olympic Games and 25 judokas (15 women and 10 men). The final sample that could be accessed was 20 athletes ( $80 \%$ of the total population) distributed as follows: women $(n=12,60 \%)$ and men $(n=8,40 \%)$. The research instrument was a semi-structured interview. The conclusions of this work highlight, first of all, the great importance of psychological aspects in the training process according to the opinion of the athletes themselves. In this sense, the most important psychological contents to reach the elite, according to the interviewees, are perseverance, constancy, the capacity for sacrifice and self-confidence. Motivation is also valued in a very necessary way, especially at the beginning to achieve practice habits and avoid premature abandonment.
\end{abstract}

Keywords: Sports training, judo, Olympic Games, psychological aspects.

Recibido: 6-nov-2019

Aceptado: 12-nov-2019

Publicado: 20-nov-2019

Como citar (apa): Robles-Rodríguez, A., Abad-Robles, M. T., Robles-Rosdríguez, J. \& Giménez, F. J. (2020). Factores psicológicos asociados a la formación y al rendimiento en judokas de elite. JUMP, (1), 27-37.

Como citar (vancouver): Robles-Rodríguez, A., Tomás Abad-Robles, M. T., Robles-Rodríguez, J. \& Giménez, F. J. Factores psicológicos asociados a la formación y al rendimiento en judokas de elite. JUMP. 2020;(1), 27-37.

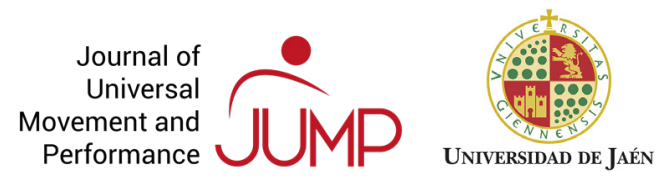




\section{Introducción}

El proceso de formación de los deportistas desde que se inician hasta que llegan a la elite va a depender de factores de tipo biológico, psicológico, perceptivo-cognitivo y social (Abernethy, Thomas y Thomas, 1993; Housner y French, 1994). Klissouras, Geladas y Koskolou, 2007) plantean que, aunque la genética y el entrenamiento establezcan los límites biofísicos del rendimiento humano, hay pruebas de que el aspecto psicológico determina en último caso el rendimiento deportivo. En este sentido, Mujika (2010) indica quelos aspectos psicológicos pueden ser determinantes a la hora de afrontar tanto el entrenamiento como la competición, sea cual sea el deporte practicado. Publicaciones recientes han establecido relaciones entre un gran número de habilidades psicológicas (autoconfianza, motivación, concentración, visualización o control de la activación, estrés y ansiedad en el deporte) y el rendimiento del propio deportista en su especialidad (Sosa, Jaenes, Godoy y Oliver, 2009; Williams, 2009). De ahí la importancia de un buen entrenamiento psicológico en el deporte de elite, donde se hace necesaria la adquisición de características psicológicas específicas (Baker y Horton, 2004). De esta manera, para Janelle y Hillman (2003), las habilidades psicológicas referidas al máximo rendimiento deben incluir atributos como: motivación, estrategias enfocadas al éxito, construcción de la confianza en sí mismo y mantenimiento de una actitud positiva, capacidad de entrenar y habilidades interpersonales.

En relación con la relevancia de estos aspectos y su aplicación directa en judo (objeto de estudio en este trabajo), se aprecian investigaciones sobre las características psicológicas como predictores del rendimiento en judokas y luchadores (LópezGullón, et al., 2011; Ruiz, 2008), estudios que analizan las características de luchadores respecto a su potencial físico (Martínez-Abellán et al., 2010), trabajos que analizan la trayectoria deportiva de los judokas de alto rendimiento desde edades tempranas (Gimeno y Guedea, 2001), y otros que indagan en la relación entre judoka y entrenador (Ruiz, 2007). Sin embargo, se detecta que hay un vacío respecto a estudios en los que se investigue sobre cómo influyen específicamente los aspectos psicológicos a lo largo del proceso de formación del joven deportista.
Por ello, el objetivo principal de este estudio fue analizar la importancia que los factores psicológicos tienen en el proceso de formación recorrido por los judokas de elite españoles que han representado a España en los Juegos Olímpicos celebrados en el siglo XXI (Sidney 2000, Atenas 2004, Pekín 2008, Londres 2012, y Rio 2016).

\section{Metodología}

La investigación se centró en aquellos judokas de elite españoles que habían participado en unos Juegos Olímpicos en el siglo XXI. Este periodo abarcó cinco Juegos Olímpicos y 25 deportistas (15 mujeres y 10 hombres). La muestra final de deportistas a la que se accedió fue de 20 judokas (80\% de la población total) distribuidos de la siguiente forma: mujeres $(n=12,60 \%)$ y hombres $(n=8,40 \%)$.

La base de datos de la Real Federación Española de Judo y Deportes Asociados facilitó el número de judokas españoles que habían sido olímpicos durante el siglo XXI. Una vez preparada la entrevista y conseguidos los datos de los judokas olímpicos participantes en el estudio, nos pusimos en contacto con cada uno de ellos para poder llevar a cabo la entrevista propiamente dicha. Cada entrevista fue transcrita literalmente y analizada con la ayuda del programa informático especializado para la investigación cualitativa MAXQDA 10. Para que el proceso de análisis fuese más fiable, la codificación se llevó a cabo por un grupo de 4 codificadores. A dicha codificación se le realizó el grado de coherencia entre expertos y el índice de Kappa de Cohen que mide el acuerdo entre las evaluaciones de dos jueces cuando ambos están valorando el mismo objeto. Tras cuatro sesiones conjuntas de codificación, se consiguió llegar al 86,76\% de acuerdo (considerado alto, lo que permitió que los miembros del grupo codificaran las entrevistas de manera individual). También en esta reunión se obtuvo un índice de Kappa bastante alto, 0,836 con $p<0,001$.

Además, con el objetivo de obtener mayor validez, todas las entrevistas de la investigación fueron repartidas entre los miembros del grupo de codificadores. Cada entrevista fue analizada por el investigador principal y por un codificador del estudio. De esta manera, cada entrevista fue codificada por dos personas. Una vez codificadas todas las entrevistas, éstas fueron comparadas y analizadas por el coordinador del estudio, con el 
fin de comprobar la coherencia entre los códigos y decidir las correcciones oportunas.

Como instrumento de investigación se diseñó una entrevista semiestructurada (Robles, Robles, Giménez y Abad, 2016). Para la validación de la misma, se llevó a cabo un análisis cualitativo y cuantitativo mediante la valoración de 10 expertos. Se analizó la validez de contenido a través del coeficiente $V$ de Aiken, estableciendo un intervalo de confianza del $99 \%$ y y el coeficiente de variación. Para conocer la fiabilidad se pasó la entrevista a judokas de alto nivel en dos momentos distintos y se analizó la consistencia interna por el método del Alfa de Cronbach $(0,915)$ y la fiabilidad test-retest utilizando el coeficiente de correlación intraclase, resultando $0,843(p<0,01)$.

\section{Resultados}

A continuación, se exponen los principales resultados obtenidos en la investigación, divididos en función de las diferentes categorías analizadas.

\section{Categoría Importancia Psicológicos \\ Aspectos}

Los 20 judokas entrevistados, opinaron que los aspectos psicológicos tenían muchísima importancia y los consideraban claves para el alto rendimiento. La gran mayoría coincidió en que, en alta competición, donde los niveles son tan semejantes, quien esté mejor psicológicamente en ese momento tiene más posibilidades de conseguir éxito. Además, estimaron clave ser fuerte psicológicamente para soportar las horas de duro entrenamiento, y la presión a la que se ven sometidos en competiciones de máxima importancia. También son importantes para estar concentrado en competición y no verse distraído por agentes externos a ella.

"El aspecto psicológico, a nivel de competición yo diría que es de lo más importante que hay. Hay mucha gente que es muy buena haciendo su deporte, pero luego psicológicamente en los momentos más duros no es capaz de soportar la presión, no es capaz de soportar los entrenamientos, entonces yo creo que la parte psicológica es muy importante, si no tienes la parte psicológica, yo creo que es bastante complicado llegar a poder hacer un deporte a alto nivel" (Sujeto 2, párrafo 29).
"Está claro que a igualdad de condiciones de dos judokas del mismo peso y mismo entrenamiento va a ganar el que mejores condiciones psicológicas tenga" (Sujeto 6, párrafo 28).

"Creo que es vital el aspecto psicológico, si tú a nivel psicológico lo tienes claro, creo que al final la cabeza lo es todo, la frase del querer es poder es así, o sea, ya no es solo por la determinación a la hora de conseguir un objetivo, sino también por aguantar la presión, al final te juegas todo a una carta, te juegas muchas horas y renuncias a muchas cosas y estas sometido a muchísima presión, entonces si eso no lo sabes manejar a nivel psicológico creo que puedes crearte una ansiedad que te perjudica para competir" (Sujeto 12, párrafo 29).

\section{Categoría Aspectos Destacados}

En esta dimensión se pretendía conocer qué aspectos psicológicos consideraban los judokas que le habían ayudado a llegar a la elite. Los entrevistados resaltaron la perseverancia $(n=8)$, la constancia $(n=7)$, la capacidad de sacrifico $(n=6)$ y la autoconfianza $(n=5)$.

"Creo que soy como muy constante, muy perseverante. Sí, soy la constancia y la perseverancia, el estar cansada y seguir, el ponerme un objetivo e intentar conseguirlo, y luego creo que soy una persona que, en momentos claves, en momentos donde me estaba jugando mucho, normalmente respondía bien, respondía mejor incluso con presión que sin presión" (sujeto 12, párrafo 30).

Tabla 1. Técnicas utilizadas para controlar las situaciones negativas en los campeonatos.

\begin{tabular}{l}
\hline Técnicas para controlar las emociones \\
\hline Confiando en el trabajo realizado y en sí mismo \\
Concentrarse en el momento, dejando de un lado las emociones \\
existentes \\
Intentando transformar lo negativo en positivo \\
Intentar disfrutar de la competición, olvidando un poco el objetivo \\
de la competición \\
Estudiando y visualizando el combate para ganar confianza \\
Darle a las derrotas la importancia justa \\
Controlar la respiración para retomar el autocontrol \\
Olvidar lo que ha salido mal y centrarse en lo que ha salido bien
\end{tabular}

Fuente: elaboración propia. 
"Pienso que se debe fundamentalmente a mi perseverancia y a mi determinación" (sujeto 16, párrafo 70).

\section{Categoría Estrategias de Entrenamiento Psicológico}

En esta categoría se analizó si los judokas entrevistados habían trabajado con psicólogos, durante qué etapa, qué importancia creían que había tenido ese trabajo con estos profesionales y de qué manera les había influido esto en sus éxitos deportivos. También se pretendía conocer qué estrategias de entrenamiento psicológico utilizaban ante situaciones negativas en los campeonatos. En cuanto al trabajo con psicólogos, la mayor parte de los sujetos entrevistados habían trabajado con estos profesionales, aunque de forma esporádica. Concretamente sólo cuatro afirmaron no haber trabajado con psicólogos (un hombre y tres mujeres). Esporádicamente lo hicieron cinco sujetos (un hombre y cuatro mujeres).

14 de los 16 judokas que habían trabajado en alguna ocasión con psicólogos, consideron que éste trabajo había sido muy importante y que había tenido gran influencia en sus éxitos deportivos. Respecto a la manera en la que influyó dicho trabajo en el rendimiento, destacaron las técnicas de relajación $(n=4)$, técnicas de visualización de combates $(n=3)$, y el trabajo sobre el aumento de la motivación $(\mathrm{n}=3)$ entre otras.

"Por supuesto me ha influido, porque me ha enseñado a tener una buena gestión de pensamientos, tener un plan de actuación, a entrenar con objetivos, a hacer estudios de rivales, y también me ha enseñado técnicas de activación y relajación. La verdad es que me ha servido muchísimo el trabajo con el psicólogo" (sujeto 12, párrafo 34).

"Yo creo que me dio las herramientas para controlar mi estrés el día del campeonato, me dio las herramientas de cómo tenía que llevar mi entrenamiento psicológico, porque yo por ejemplo me leía todos los libros de autoayuda, de entrenamiento mental, todos los que caían en mis manos me los leía, pero a veces tú te lees todos esos libros, pero no sabes cómo hacer una programación de entrenamiento psicológico, entonces ahí sí que me ayudó mucho el psicólogo" (sujeto 18, párrafo 34).
Para terminar con esta categoría se quiso conocer si los judokas controlaban las emociones ante situaciones negativas en los campeonatos, a lo que todos los entrevistados contestaron que sí $(n=20)$. Todas las técnicas que utilizaban para controlarlas iban encaminadas a reducir la presión existente en ese momento y a ganar confianza en sí mismo (Tabla 1).

\section{Categoría Responsable Preparación Psicológica}

En esta categoría se indagó sobre quién había sido el responsable de la preparación psicológica del judoka. Cabe destacar que todos los entrevistados comenzaron con el trabajo psicológico una vez que ya estaban en la elite. Por otro lado, la mayoría realizaron trabajo psicológico específico en momentos concretos de su carrera deportiva como ya se detalló anteriormente. Concretamente, 13 de los entrevistados afirmaron que el responsable de su preparación psicológica fue el psicólogo deportivo, cuatro sujetos señalaron que los entrenadores fueron los que se encargaron de ese trabajo psicológico, dos sujetos indicaron que ellos mismos, y uno, un psicólogo clínico.

\section{Categoría Emociones}

Esta categoría analizó las sensaciones de los judokas durante los campeonatos, así como su estado emocional, tanto en los días previos a las competiciones, como en el día de las mismas. En lo que a las sensaciones se refiere, hay judokas en los que las sensaciones en los campeonatos nacionales eran distintas a los internacionales, debido a la presión que sentían por ganar siempre que competían en campeonatos de España, por lo que el nerviosismo era mucho mayor.

"Bueno, las sensaciones son distintas. En un campeonato de España vas con la obligación de ganar obligatoriamente, y en los campeonatos internacionales sabes que hay más gente que te puede ganar. $Y$ bueno también hay campeonatos en los que no te juegas nada y vas fuera de temporada y sin jugarte puntos, hay otros que en los que te juegas puntos para los JJ.00. o para clasificarte para el campeonato del mundo como ha pasado algunas veces" (Sujeto 6, párrafo 32).

"Pues en los nacionales tenía un punto de 
nerviosismo porque al final es donde te juegas todo, el papel que necesitas para luego poder salir, entonces sí que hay un punto de presión, y en cambio, fuera he competido siempre muy tranquila, nunca he sentido el mínimo nerviosismo, si el punto de nerviosismo por las ganas de competir, pero no presión, ha sido bastante distinto" (Sujeto 9, párrafo 37).

En cuanto al estado emocional previo a los días de la competición, este variaba dependiendo de las circunstancias de cada judoka. Se detectó que el estado emocional dependía de muchos factores y diferentes estados de ánimos entre los que destacaron la sensación de tranquilidad $(n=7)$, concentración $(n=6)$, nerviosismo $(n=4)$ y tensión o ansiedad $(n=4)$, entre otros.

El día de la competición, el estado emocional era parecido en muchos de los judokas entrevistados, girando en torno a la concentración $(n=11)$, nerviosismo $(n=9)$ y motivación $(n=6)$, con alguna excepción.

\section{Categoría Motivación}

En esta categoría se pretendía conocer el grado de motivación de los judokas durante los entrenamientos y campeonatos. Lo primero que hay que decir es que los sujetos entrevistados creían esencial que durante el proceso de formación deportiva los judokas fueran motivados, lo que facilitaría que el niño no abandonara la práctica deportiva, y que no se perdiesen tantos deportistas por el camino, si no llegan a la elite, por lo menos que sigan practicando judo.

Otra cuestión es cuando los judokas llegan a la elite, donde el grado de motivación puede variar. En el caso de los deportistas entrevistados, este varió durante los entrenamientos entre alto y muy alto. Los judokas entrevistados que tenían el grado de motivación alto o muy alto, era porque lo que les gustaba principalmente era hacer judo, y lo que les movía principalmente era el amor y el disfrute por este deporte, como nos cuentan por ejemplo el sujeto 17.

"Normalmente bien, aparte de por competición a mí me gusta mucho hacer judo, entonces era fácil motivarme en los entrenamientos, siempre hay etapas en las que estas más cansada o te cuesta más llevarlo, pero normalmente mi grado de motivación era bueno" (Sujeto 17, párrafo 39).

Por otro lado, estaban los que sufrían altibajos en el grado de motivación. Este variaba normalmente con las sensaciones obtenidas durante el entrenamiento. Si las sensaciones eran buenas, el grado de motivación era alto y viceversa. Aquí también se daba otro caso, que es el de los pesos pesados, su grado de motivación iba acorde al número de compañeros que tenían para entrenar, ya que era difícil encontrar gente pesada para conseguir un buen entrenamiento. En este caso, el nivel de motivación se relacionaba con el número de compañeros que tenían para poder entrenar bien.

"Muy grande, pero también había entrenamientos que lloraba porque no me salían las cosas y perdía la confianza, que la tenía que recuperar para el día siguiente, pero como siempre tenía un objetivo esa era la motivación para entrenar más" (Sujeto 20, párrafo 37)

"A veces bastante bajo por no tener gente, pero bueno siempre buscábamos soluciones y trabajo para ir remediando esa situación. El no tener gente es un problema que tienen todos los pesos pesados y en España es más grave todavía porque como hay menos medios y tal... pero bueno, lo remediábamos como podíamos" (Sujeto 6, párrafo 35).

En lo que respecta al grado de motivación durante los campeonatos, este era máximo en todos los campeonatos, ya que estos servían como herramientas de motivación para los jóvenes. En relación con la etapa de alto rendimiento, 18 de los 20 judokas entrevistados, consideraron que tenían un grado de motivación alto o muy alto durante los campeonatos, ya que como ellos mismos decían, era para lo que realmente entrenaban. Solo se encontraron dos judokas, uno masculino y una fémina, en los que el nivel de motivación variaba, según los resultados obtenidos en los campeonatos anteriores, a buenos resultados, alto grado de motivación, y a malos resultados, bajo grado de motivación. Además, un judoka masculino y tres femeninas, consideraron que la motivación tenía una relación directa con la confianza, a mayor grado de motivación mayor confianza, y viceversa. 


\section{Categoría Autoconfianza}

En esta categoría se intentaba conocer el nivel de autoconfianza que tenían los judokas durante los campeonatos y la manera en la que creían que influía este aspecto en los resultados deportivos. La gran mayoría de los entrevistados afirmaron que su autoconfianza había sido o era alta, como muestran los sujetos 8 y 12 .

"Mi confianza era muy alta, además pienso que esto es muy importante, porque si piensas que vas a ganar haces buen combate, pero si piensas que el adversario es fuerte y es mucho para mí, ¿cómo vas a ganar?" (Sujeto 8, párrafo 45).

"Durantemuchosañosminivel deautoconfianza era altísimo, yo confiaba totalmente en mí, creo que es importantísimo además para ganar un combate. Si tu nivel de autoconfianza no está al $100 \%$, lo normal es que no ganes los combates" (Sujeto 12, párrafo 42).

Delos 20 sujetos entrevistados, 19 consideraron muy importante que la autoconfianza fuera alta, ya que esto les ayudaba a sentir que podían ganar a cualquier oponente, lo que conlleva una mejora en el rendimiento y a su vez la consecución de buenos resultados. Dos judokas femeninas reconocían que su nivel de autoconfianza mejoró con la ayuda del psicólogo. Además, un judoka masculino y tres femeninas, estimaron que la confianza tenía una relación directa con motivación, a mayor grado de motivación, mayor confianza, y viceversa, como vimos en la categoría anterior. Por otro lado, un judoka consideró que el judoka español, en general, tenía poca confianza en sí mismo a la hora de competir internacionalmente. También cabe destacar, que tres judokas femeninas pensaban que la confianza había que controlarla, ya que un alto nivel de ésta puede llevar a un exceso de la misma, lo que puede provocar una pérdida de tensión y su correspondiente derrota en el combate. Por último, la judoka que decía tener un bajo nivel de confianza, le encuentra su parte positiva, ya que, obliga a no confiarte con nadie y a salir a ganar siempre.

\section{Categoría Presión/Estrés}

Con esta categoría se indagaba sobre si se habían sentido presionados ante la consecución de determinados objetivos, y de qué manera habían afrontado dicha presión. En este apartado cabe destacar que la gran mayoría de los sujetos, donde mayor presión sentían era en los campeonatos de España, debido a la obligación que tenían de ganar para salir al circuito internacional, y para mantener su "status". De los 20 sujetos entrevistados, 13 estimaban que la presión ante determinados objetivos les había influido negativamente en alguna ocasión, destacando la sensación de nerviosismo, lo que se transformaba en ansiedad y provocaba un bloqueo, lo cual afectaba al rendimiento. Para evitar que esto se siguiera repitiendo, buscaban diferentes herramientas para bajar el foco de presión y poder rendir mejor. Éstas consistían en intentar buscarle siempre el lado positivo a las derrotas y restarle importancia a la competición, lo cual reduciría el foco de presión que ejerce la misma. Por el contrario, siete judokas (cuatro hombres y tres mujeres) consideraron que nunca la presión les influyó de forma negativa, y que ésta les ayudaba a rendir mejor, que se crecían bajo situaciones de presión.

\section{Categoría Principales Psicológicos en Competición}

Anteriormente se analizó qué aspectos psicológicos consideraban los judokas que le habían ayudado a llegar a la elite, mientras que en esta categoría lo que se pretendía es conocer los principales aspectos psicológicos que le habían ayudado a rendir al máximo en alta competición. Como ocurrió en la categoría aspectos psicológicos destacados, todos los judokas entrevistados resaltaron más de un aspecto, como por ejemplo los sujetos 1, 10 y 16 (ver Tabla 2).

\section{Categoría Rendimiento}

En cuanto al rendimiento de unos judokas sobre otros, la mayoría de los sujetos entrevistados relacionaban esta falta de rendimiento con cuestiones de confianza y de no saber afrontar la presión de manera adecuada. Se encuentran tres judokas masculinos que no sabrían qué contestar sobre este tema, ya que a su juicio el rendimiento dependía del carácter de cada persona; y por otro lado existían dos judokas femeninas que achacaban esta falta de rendimiento a cuestiones técnico-tácticas, y no solo a factores psicológicos (Tabla 3). 
Tabla 2. Principales aspectos psicológicos que les han ayudado a rendir al máximo en la elite.

\begin{tabular}{llll}
\hline Principales Aspectos Psicológicos en Competición & Hombres & Mujeres & Total \\
\hline Constancia / Perseverancia & 1 & 5 & 5 \\
Autoconfianza & 2 & 3 & 4 \\
Espíritu/Capacidad de sacrificio & 1 & 3 & 2 \\
Alto grado de concentración & 1 & 1 & 2 \\
Ambición & 0 & 1 & 1 \\
Carácter positivo & 0 & 1 & 1 \\
Carácter luchador & 1 & 0 & 1 \\
Buena gestión del tiempo & 0 & 1 & 1 \\
Motivación & 1 & 0 & 1 \\
Afán de superación & 1 & 0 & 1 \\
Adecuada toma de decisiones & 1 & 0 & 1 \\
Estabilidad en la victoria y en la derrota & 1 & 0 & 1 \\
Alta capacidad de aprendizaje & 0 & 1 \\
Amor Propio & & 1 \\
\hline Fuente elaborán propia & & 1 \\
\hline
\end{tabular}

Fuente: elaboración propia.

Tabla 3. Motivos por los que, a nivel psicológico, unos judokas rendían más que otros.

\begin{tabular}{llll}
\hline Motivos & Hombres & Mujeres & Total \\
\hline No son capaces de gestionar la presión/estrés, por lo que no rinden al máximo en & 1 & 9 & 10 \\
este tipo de situaciones & 2 & 1 & 3 \\
Falta de autoconfianza & 1 & 0 & 1 \\
Exceso de confianza & 1 & 0 & 1 \\
Mala autoevaluación de la confianza & & \\
\hline
\end{tabular}

Fuente: elaboración propia.

\section{Discusión}

Los aspectos psicológicos son un factor determinante para los judokas olímpicos, quienes llegan a considerar que en determinados momentos quien esté mejor psicológicamente tendrá más posibilidades de obtener un mejor resultado. Además, consideran clave ser fuerte psicológicamente para soportar las altas horas de duro entrenamiento y la presión a la que se ven sometidos en competiciones de máxima importancia. En esta línea nos encontramos estudios que indicaron que los factores psicológicos eran los más importantes para el desarrollo y logro del desempeño de excelencia deportiva (Ferreira, Gomes, Borges, Routen y Almeida, 2015; Klissouras et al., 2007; MacNamara, Button y Collins, 2010; Ruiz, Sánchez, M., Durán y Jiménez, 2006).

Según los datos obtenidos, los entrevistados aluden a la adquisición de determinados aspectos psicológicos individuales como claves para la consecución de resultados. Entre ellos destacan: perseverancia, constancia, capacidad de sacrificio, autoconfianza, positividad, disciplina, autodeterminación, afán de superación, mentalidad ganadora, concentración, motivación, capacidad de visualizar, adecuada toma de decisiones, gestión del tiempo y control de la presión, lo cual coincide con otras investigaciones (Buceta, 1998; Cárdenas, 2003; Escarti, Pacual y Gutiérrez, 2005; Ferreira et al., 2015; Hellison, 1995; Sánchez, 2002).

A pesar de que todos los judokas consideran imprescindible el entrenamiento de los aspectos psicológicos para el máximo rendimiento deportivo, solo algunos deportistas han llevado un entrenamiento psicológico continuado, y la mayoría únicamente han trabajado estos aspectos durante momentos puntuales de su preparación. Este trabajo psicológico se produce principalmente ante una crisis concreta o antes de una competición importante, al igual que los resultados encontrados en los trabajos realizados por Gordin (1995) y Balague (2005).

Coincidiendo con varios estudios (Martín, 2006; Morilla y Pérez, 2004; Olmedilla, 2001; Sosa et al., 2009; Williams, 2009) los judokas entrevistados realizan distintas técnicas de trabajo psicológico 
ante situaciones complicadas o de máxima exigencia, sobre todo cuando el resultado obtenido no ha sido el esperado. Para ello, utilizan estrategias encaminadas a recuperar la autoconfianza, mantener la concentración durante las competiciones, transformar lo negativo en positivo, buscar el disfrute durante el torneo, visualizar los siguientes combates, control de la respiración para retomar el autoncontrol y reforzar todo aquello que esté saliendo bien.

Se observa en esta investigación, que los judokas que han realizado entrenamiento psicológico, ya sea de manera habitual o esporádicamente, quedaron muy satisfechos con los resultados obtenidos, ya que esto les ayudó a mejorar el rendimiento. Estos resultados coinciden con los obtenidos en otros estudios realizados con deportistas olímpicos (Martín, 2006) y con el realizado con judokas noruegos (Kristiansen y Roberts, 2010).

Aunque casi todos los judokas han realizado trabajo psicológico, no todos lo han hecho con un profesional de la psicología deportiva, sino que, en determinados casos, es el propio entrenador de judo quien se ocupa de este tipo de función, lo que hace necesario que él mismo adquiera nociones de entrenamiento psicológico, lo cual coincide, en este sentido, con lo hallado en otros trabajos (Acosta, 2012; Buceta, 1998, 1999; Capetillo, 2011; Contreras, Rojas y Palomino, 2012). En esta línea, Mahamud, Tuero y Márquez (2005) plantean que esto puede deberse a que son escasos los clubes, delegaciones provinciales, o federaciones regionales que cuentan con especialistas que asesoren en cuestiones psicológicas a sus deportistas.

Por otro lado, un factor importante en un deporte como el judo, es el estado emocional y las sensaciones de los judokas en los días previos a las competiciones. En esta investigación suelen variar dependiendo de las circunstancias de cada judoka. Normalmente oscila entre nerviosismo, ansiedad, tensión, preocupación, irritabilidad, angustia, concentración, o motivación, coincidiendo con lo que establecen otros estudios (Grills y Ollendick, 2002; Storch, Storch, y Killiany, 2005). Estos estados emocionales o sensaciones antes de las competiciones, aunque en un principio puedan parecer negativos, a los deportistas de alto rendimiento les puede ayudar a aumentar las probabilidades de éxito, puesto que existen estudios (Hammermeister y Burton,
1995; Lane, Terry, y Karageorghis, 1995; Weinberg y Gould, 2010) que demostraron que la ansiedad precompetitiva no reduce el rendimiento de los deportistas. Además, suele variar el día de la competición, y las sensaciones de los días previos se transforman en tranquilidad, motivación, concentración y activación principalmente, lo cual se observa en este estudio.

Uno de los aspectos psicológicos que más se ha destacado en la investigación es la motivación, ya que de ella va a depender en gran medida la continuación del joven deportista. Coincidiendo con lo que dicen varios estudios (Hansen, Larson, y Dworkin, 2003; Kirk, 2005; Piñar y Cárdenas, 2010) la participación en actividades lúdicas y motivadoras en los entrenamientos de judo, en distintas competiciones a nivel formativo (sin exigencia de resultados) y el compaginar varias actividades deportivas, es lo que permite que, durante las primeras etapas de la formación deportiva, la motivación se mantenga siempre alta. Sin embargo, durante la etapa de alto rendimiento influyen otros factores para que la motivación sea siempre alta. En concreto, en este trabajo se observa cómo la motivación ha ido en consonancia con la autoconfianza. Por norma general, los judokas siempre han mantenido la motivación alta y con ello la autoconfianza, pero en ocasiones aparecen determinados aspectos que provocan un descenso en ambas. Coincidiendo con Suay, Salvador y Ricarte (1998), Kentta (2001) el sobreentrenamiento es una causa de desmotivación y disminución del rendimiento, por lo que es importante que exista una dosificación de las cargas en los deportistas de elite. También hay que destacar que la principal causa por la que la motivación y autoconfianza alcanzan altos niveles es la consecución de resultados o de los objetivos establecidos, como así se ve reflejado en numerosas investigaciones (Guillén y Álvarez-Malé, 2010; Jaenes, Peñalosa, Navarrete y Bohórquez, 2011, 2012; Sosa et al., 2009; Williams, 2009).

En este trabajo se observa un dato importante del que no hemos encontrado referencias en otros estudios. Se trata de la principal causa de desmotivación de los pesos pesados, quienes suelen sufrir estados de baja motivación a causa de no encontrar suficientes compañeros de características similares para poder realizar entrenamientos de calidad que le permitan mejorar el rendimiento. 
Finalmente, cabe destacar que un aspecto clave para tener éxito en la alta competición es el saber gestionar la presión ante determinados objetivos. Se observa que en mayor o menor medida los judokas entrevistados han sabido gestionar esta presión, lo que le ha permitido llegar y mantenerse en la elite deportiva. En este sentido, existen investigaciones que demostraron que soportar situaciones potencialmente estresantes y saber gestionar la presión es garantía de éxito (Buceta 1998, 1999; Hernández, 2007; Olmedilla, 2005; Sánchez y Torregrosa, 2005). En relación con lo anteriormente expuesto, los judokas entrevistados, creen que es precisamente el no saber gestionar esa presión y no tener altos niveles de autoconfianza y motivación es lo que no permite a muchos judokas rendir al máximo y llegar a la elite deportiva.

\section{Conclusiones}

El objetivo principal de este estudio fue analizar la importancia que los factores psicológicos tienen en el proceso formativo recorrido por los judokas de elite españoles que han representado a España en los Juegos Olímpicos celebrados en el siglo XXI (Sidney 2000, Atenas 2004, Pekín 2008, Londres 2012, y Rio 2016). En este sentido, como principales conclusiones de este trabajo se puede destacar, en primer lugar, la gran importancia de los aspectos psicológicos en el proceso formativo según la opinión de los propios deportistas; tanto para poder aguantar el ritmo de los entrenamientos como para poder competir en alta competición. Además, en cuanto a los factores psicológicos más importantes para llegar a la elite, los entrevistados resaltaron la perseverancia, la constancia, la capacidad de sacrifico y la autoconfianza. Respecto a la manera en la que influyó dicho trabajo en el rendimiento, destacan las técnicas de relajación, técnicas de visualización de combates, y el trabajo sobre el aumento de la motivación entre otras. También cabe resaltar que el estado emocional de estos deportistas depende de muchos factores y diferentes estados de ánimos entre los que destacaron la sensación de tranquilidad, concentración, nerviosismo y tensión o ansiedad, entre otros.

Por último, la motivación es valorada de forma muy necesaria, sobre todo en los comienzos para conseguir hábitos de práctica y evitar abandonos prematuros. Unida a la motivación, la autoconfianza es una cualidad destacada también por la mayoría para poder llegar a la elite deportiva.

Las limitaciones del estudio estuvieron relacionadas con la inexistencia de este tipo de trabajos en Judo. Además, gran parte delos judokas ya se encontraban retirados y desvinculados del ámbito federativo, por lo que fue difícil encontrar un lugar (competiciones, concentraciones...) en el que reunirlos con el fin de poder llevar a cabo las entrevistas. Futuras investigaciones podrían estudiar el proceso de formación de los judokas Olímpicos que participen en Juegos Olímpicos celebrados posteriormente al estudio realizado y ver similitudes y diferencias con la investigación realizada. También, se podrían realizar de estudios de casos, y entrevistar a familiares, amigos y entrenadores, con el fin de profundizar aún más.

\section{Aplicaciones prácticas}

De los hallazgos encontrados en el estudio presentado, parece conveniente aplicar estrategias, desde las primeras etapas, dirigidas al mantenimiento de la motivación de los deportistas, Para ello, es importante diseñar entrenamientos de calidad que fomenten una alta participación e implicación de cada deportista. Además, también se plantea la necesidad de incluir la competición en el entrenamiento de forma progresiva, realizando competiciones adaptadas a la edad y al nivel de los judokas. Se debería evitar también una selección demasiado temprana de jóvenes talentos, ya que esto puede disminuir la motivación de los "no seleccionados", pudiendo llegar incluso a un abandono de la práctica deportiva, lo cual, a su vez, también implica la ausencia de búsqueda de resultados en edades tempranas y a corto plazo. Finalmente, también parece necesario planificar los entrenamientos de forma integral, incluyendo los contenidos psicológicos como un contenido necesario más de los mismos. En este sentido, sería conveniente plantear programas de formación continua para entrenadores que les ayuden a mejorar dichas planificaciones y a aumentar sus conocimientos sobre psicología del deporte. 


\section{Referencias}

Abernethy, B., Thomas, K. T. \& Thomas, J. T. (1993). Strategies for improving understanding of motor expertise (or mistakes we have made and things we have learned!). En J. L. Starkesm y F. Allard (Eds.), Cognitive issues in motor expertise (pp. 317-356). Amsterdam: Elsevier Science.

Acosta, E. (2012). Iniciación y Formación Deportiva: Una reflexión siempre oportuna. U.D.C.A. Actualidad y Divulgación Científica, $15,57-65$

Baker, J. \& Hortonb, S. (2004). A review of primary and secondary influences on sport expertise. High Ability Studies, 15, 211-228.

Balague, G. (2005). Periodization of Psychological Skills Training: Preparing for the Horizontal Jumps. En R. Vernacchia y T. Statler (Eds.), The Psychology of High-Performance Track and Field. Mountain View, CA: Tafnews Press.

Buceta, J. M. (1998). Psicología del entrenamiento deportivo. Madrid: Dykinson.

Buceta, J. M. (1999). Intervención psicológica en el entrenamiento deportivo: estrategias para optimizar el funcionamiento de jugadores de baloncesto en sesiones de ensayo repetitivo. Revista de Psicología del Deporte, 8, 39-52.

Capetillo, R. (2011). Factores sociales que influyen en la formación deportiva de adolescentes. Revista Lúdica Pedagógica, 16(2), 39-48.

Cárdenas, D. (2003). El proceso de formación táctica desde una perspectiva constructivista. En A. López, C. Jiménez y R. Aguado (Eds.), Didáctica del baloncesto en etapas de iniciación. Madrid: Fundación Real Madrid.

Contreras, D., Rojas, D. \& Palomino, O. (2012). Efectos de dos formas de periodizar la carga (lineal y doble ondulada) en el entrenamiento de la fuerza, sobre la cinemática de la salida estática voluntaria en patinadores de carreras pre púberes. Revista Actividad Física y Desarrollo Humano, 4(1), 89-96.

Escarti, A., Pascual, C. \& Gutiérrez, M. (2005). Responsabilidad personal y social a través de la educación física y el deporte. Barcelona: Grao.

Ferreira, T., Gomes, J. C., Borges, H., Routen, A., y Almeida, A. (2015). Elite coach's views on factors contributing to excellence in orienteering. Cultura, Ciencia y Deporte, 10, 77-86.

Gimeno, F. \& Guedea, J. A. (2001). Evaluación e intervención psicológica en la «promoción" de talentos deportivos en judo. Revista de Psicología del Deporte, 10(1), 103-125.

Gordin, R. D. (1995). Hypnosis in Sports. En P. Keith y F. William (Eds.), Sport Psychology an Analysis of Athlete Behavior (3a ed., pp. 193-201). Longmeadow: Mouvement Publications.

Grills, A. \& Ollendick, T. (2002). Peer Victimization, Global SelfWorth, and Anxiety in Middle School Children. Journal of Clinical Child and Adolescent Psychology, 37(1), 59-68.

Guillén, F. \& Álvarez-Malé, M. L. (2010). Relación entre los motivos de la práctica y la ansiedad en jóvenes nadadores de competición. Revista Iberoamericana de Psicología del Ejercicio y el Deporte, 5(2), 233-252.

Hammermeister, J. \& Burton, D. (1995). Anxiety and the Ironman - investigating the antecedents and consequences. Sport Psychologist, 9(1), 29-40.

Hansen, D. M., Larson, R. W. \& Dworkin, J. B. (2003). What adolescents learn in organized youth activities: A survey of self-reported developmental experiences. Journal of Research on Adolescence, 13, 25-55

Hellison, D. (1995). Teaching personal and social responsibility through physical education. Champaign, IL: Human Kinetics.

Hernández, J. G. (2007). Herramientas aplicadas al desarrollo de la concentración en el alto rendimiento deportivo. Cuadernos de Psicología del Deporte, 7(1), 61-70.

Housner, L. D. \& French, K. E. (1994). Future directions for research on expertise in learning, performance, and instruction in sport and physical activity. Quest, 46, 241-246.
Jaenes, J. C., Peñalosa, R., Navarrete, K. G. \& Bohórquez, M. R. (2011). Ansiedad y autoconfianza precompetitiva en maratonianos y triatletas. Revista Internacional de Ciencias Sociales y Humanidades, SOCIOTAM, 21(2), 95-110.

Jaenes, J. C., Peñalosa, R., Navarrete, K. G. \& Bohórquez, M. R. (2012). Ansiedad y autoconfianza precompetitiva en triatletas. Revista Iberoamericana de Psicología del Ejercicio y el Deporte, 7(1), 113-124

Janelle, C. M. \& Hillman, C. H. (2003). Expert performance in sport: Current perspectives and critical issues. En J. L. Starkes y A. Ericsson (Eds.), Expert Performance in Sport (pp. 19-47). Champaign, IL: Human Kinetics.

Kentta, G. (2001). Trainning practices and over training syndrome in Swedis age-group athletes. International Journal of Sports Medicine, 22, 460-465.

Kirk, D. (2005). Physical education, youth sport and lifelong participation: The importance of early learning experiences. European Physical Education Review, 11, 239-255.

Klissouras, V., Geladas, N. \& Koskolou, M. (2007). Nature prevails over nurture. International Journal Sport Psychology, 38, 3567.

Kristiansen, E. \& Roberts, G. C. (2010). Young elite athletes and social support: coping with competitive and organizational stress in "Olympic" competition. Scandinavian Journal of Medicine and Science in Sport, 20, 686-695.

Lane, A. M., Terry, P. C. \& Karageorghis, C. I. (1995). Path analysis examining relationships among antecedents of anxiety, multidimensiona state anxiety and triathlon performance. Perceptual and Motor Skills, 81(3), 1255-1266.

López-Gullón, J. M., García-Pallarés, J., Berengüi, R., MartínezMoreno, A., Morales, V. \& Torres-Bonete, M. D. (2011). Factores físicos y psicológicos predictores del éxito en lucha olímpica. Revista de Psicología del Deporte, 20(2), 573-588.

MacNamara, A., Button, A. \& Collins, D. (2010). The role of psychological characteristics in falicitating the pathway to elite performance part 1: Identifying mental skills and behaviors. The Sport Psychologist, 24, 52-73.

Mahamud, J., Tuero, C. \& Márquez, S. (2005). Características psicológicas relacionadas con el rendimiento: comparación entre los requerimientos de los entrenadores y la percepción de los deportistas. Revista de Psicología del Deporte, 14(2), 237-251.

Martín, M. (2006). Suporte psicológico aos atletas brasileiros durante as Olimpíadas de Atenas 2004. Revista Brasileira de Educação Física e Esporte, 20(5), 165-167.

Martínez-Abellán, A., García-Pallarés, J., López-Gullón, J. M., Muriel, X., Morales, V. \& Martínez-Moreno, A. (2010). Factores anaeróbicos predictores del éxito en lucha olímpica. Cuadernos de Psicología del Deporte, 10(supl.), 17-23.

Morilla, M. \& Pérez, E. (2004). Entrenamiento de la atención y la concentración. Ejercicios para su mejora. Sevilla: Difusión Gráfica.

Mujika, I. (2010). Intense training: the key to optimal performance before and during the taper. Scandinavian Journal of Medicine \& Science in Sports., 2, 24-31.

Olmedilla, A. (2001). Estrategias ambientales para el control de la atención: preparación específica del partido de fútbol. Ponencia presentada en el VIII Congreso Nacional den Psicología de la Actividad Física y el Deporte, Pontevedra.

Olmedilla, A. (2005). Factores psicológicos y lesiones en futbolistas: un estudio correlacional. Murcia: Quaderna Editorial.

Piñar, M. I. \& Cárdenas, D. (2010). La competición como herramienta formativa. Diferentes propuestas en minibasket. Wanceulen E.F. Digital, 7.

Robles, A., Robles, J., Giménez, F.J. \& Abad, M.T. (2016). Validación de una entrevista para estudiar el proceso formativo de judokas de elite. Revista Internacional de Medicina y Ciencias de la Actividad Física y el Deporte, 16(64), 723-738.

Ruiz, L., Sánchez, M., Durán, J. \& Jiménez, C. (2006). Los expertos 
en el deporte: Su estudio y análisis desde una perspectiva psicológica. Anales de Psicología, 22(1), 132-142.

Ruiz, R. (2007). Características de liderazgo en el deporte del judo. Revista de Psicología del Deporte, 16(1), 9-24.

Ruiz, R. (2008). Aportaciones del análisis subdimensional del cuestionario de personalidad BFQ para la predicción del rendimiento en judokas jóvenes de competición. Cuadernos de Psicología del Deporte, 8(1), 5-29.

Sánchez, X. \& Torregrosa, M. (2005). El papel de los factores psicológicos en la escalada deportiva: un análisis cualitativo. Revista de Psicología del Deporte, 14, 177-194.

Sánchez, M. (2002). El proceso de llegar a ser experto en baloncesto: Un enfoque psicosocial. Tesis Doctoral. Universidad de Castilla-La Mancha, Toledo.
Sosa, P. I., Jaenes, J. C., Godoy, D. \& Oliver, J. F. (2009). Variables psicológicas en el deporte. Sevilla: Wanceulen.

Storch, E., Storch, J. \& Killiany, E. (2005). Self-Reported Psychopathology in Athletes: A Comparison of Intercollegiate Student-Athletes and Non-Athletes. Joumal of Sport Behavior, 28(1), 86-98.

Suay, F., Salvador, A. \& Ricarte, J. (1998). Indicadores psicológicos de sobreentrenamiento y agotamiento. Revista de Psicología del Deporte, 13, 7-25

Weinberg, R. S. \& Gould, D. (2010). Fundamentos de psicología del deporte y el ejercicio físico ( $4^{\mathrm{a}}$ ed.). Madrid: Editorial Médica Panamericana.

Williams, J. M. (2009). Applied sport psychology: Personal growth to peak performance. Nueva York: McGraw-Hill. 